\title{
Sinus Floor Augmentation by Intraoral Endoscopic Approach for Biomaterial Study in a Rabbit Model
}

\author{
IVAN VALDIVIA-GANDUR ${ }^{1}$, VÍCTOR BELTRÁN ${ }^{2}$, EDUARDO BORIE $^{2}$, WILSON ASTUDILLO-ROZAS $^{1,3}$, \\ OSCAR ACEITUNO-ANTEZANA ${ }^{3,4}$, NICOLAS FERRER-VALDIVIA $^{3,4}$ and WILFRIED ENGELKE ${ }^{5}$ \\ ${ }^{1}$ Biomedical Department, Universidad de Antofagasta, Antofagasta, Chile; \\ ${ }^{2}$ Dental School, Clinical Investigation and Dental Innovation Center (CIDIC), Universidad de La Frontera, Temuco, Chile; \\ ${ }^{3}$ Master of Biomedical Sciences, Universidad de Antofagasta, Antofagasta, Chile; \\ ${ }^{4}$ Dentistry Department, Universidad de Antofagasta, Antofagasta, Chile; \\ ${ }^{5}$ Department of Oral and Maxillofacial Surgery, Center for Dentistry, \\ Oral Medicine and Craniomaxillofacial Surgery, University of Göttingen, Göttingen, Germany
}

\begin{abstract}
Aim: To develop a new surgical model for sinus floor augmentation (SFA) in rabbit for experimental purposes. Materials and Methods: Eight adult rabbits were used, two for a surgical design using the anatomical dissection study, and the other six for an endoscopically assisted intraoral approach of SFA unilaterally, creating a subantral space where an allograft biomaterial was deposited. SFA was verified through cone-beam computerized tomography. Healing, weight, food, feces, and behavior were evaluated for 4 weeks post-operatively. Results: All animals survived. There was no bleeding or infection; inflammation was mild. No changes were observed in terms of feeding, weight, feces, or behavior. Tissue healing was normal. Conclusion: This model is a refinement of the experimental technique and is a real option for SFA, without compromising animal morbidity because of its conservative design. The minimally invasive approach with endoscopic assistance reduces bias and improves surgical predictability.
\end{abstract}

Sinus floor augmentation (SFA) is a useful technique for treating severe sinus pneumatization before the placement of dental implants. The singular anatomy and physiology of this anatomical site, in addition to the clinical importance that it has acquired, makes it ideal for investigating biomaterials, which has led to the proposal of various animal models including the rabbit (1-3).

This article is freely accessible online.

Correspondence to: Victor Beltrán Varas, Dental School, Clinical Investigation and Dental Innovation Center (CIDIC), Universidad de La Frontera, Temuco, Chile. E-mail: victor.beltran@ ufrontera.cl

Key Words: Sinus floor augmentation, bone regeneration, endoscopy, animal models.
The maxillary sinus morphology of the rabbit is comparable to that of humans (4), and therefore, this animal model has been proven to be suitable for separation procedures of maxillary sinus mucosa from the bone, for testing graft biomaterials in evaluating new bone formation $(1,3,5)$. The experimental surgical design reported in the literature to access in the rabbit maxillary sinus describes an extraoral approach through the skin of the nasal dorsum $(3,4,6,7)$ or the masseter area (8). These kinds of procedures may have some disadvantages related to the damage of several tissue planes, increasing animal morbidity, besides questioning whether sinus floor elevation is actually achieved because access is realized extraorally, from the lateral and upper region of the maxillary sinus.

Considering these aspects, the aim of this study was to describe a novel surgical protocol of anatomical access for maxillary sinus lift in rabbits through an intraoral approach that is minimally invasive using endoscopic assistance.

\section{Materials and Methods}

The protocol was approved by the Ethics Committee of the University of La Frontera (246/006). Eight adult Californian rabbits (Oryctolagus cuniculus) were selected from the animal facility laboratory of the Department of Basic Sciences, University of La Frontera, Chile. In order to minimize growth effects, only adult animals ( 7 months old, with a weight of $2.90 \pm 0.20 \mathrm{~kg}$ ) were used in the study. The care and management of the animals were according to the ethical standards of the Guide for the Care and Use of Laboratory Animals (9), and the surgery took place in a sterile environment. Four female and four male rabbits were divided into two groups: Two specimens (one male and one female) were used to perform the surgical design through anatomical dissection; the other six rabbits (three male and three female) were used for the application and evaluation of the in vivo-designed protocol. 
in vivo $33: 1843-1849(2019)$

Table I. Rabbit Grimace Scale $(11,12)$ registered during 30 days after experimental sinus floor augmentation. 'Nose shape' was not included because objective recording was difficult for the examiner.

\begin{tabular}{|c|c|c|c|c|c|c|c|c|c|c|c|c|}
\hline \multirow[b]{2}{*}{ Rabbit no } & \multicolumn{3}{|c|}{ Orbital tightening ${ }^{\text {a }}$ (days) } & \multicolumn{3}{|c|}{ Cheek flattening ${ }^{\mathrm{b}}$ (days) } & \multicolumn{3}{|c|}{ Whisker position $^{\mathrm{c}}$ (days) } & \multicolumn{3}{|c|}{ Ear position $^{\mathrm{d}}$ (days) } \\
\hline & $\mathrm{OP}$ & MP & $\mathrm{NP}$ & $\mathrm{OP}$ & MP & $\mathrm{NP}$ & $\mathrm{OP}$ & MP & NP & $\mathrm{OP}$ & MP & NP \\
\hline 1 (Male) & $1-2$ & 3 & $4-30$ & 1 & $2-4$ & $5-30$ & $1-3$ & $4-5$ & $6-30$ & $1-2$ & $3-4$ & $5-30$ \\
\hline 2 (Male) & 1 & $2-4$ & $5-30$ & 1 & $2-3$ & $4-30$ & $1-2$ & $3-4$ & $5-30$ & 1 & $2-4$ & $5-30$ \\
\hline 3 (Male) & 1 & $2-5$ & $6-30$ & $1-2$ & $3-4$ & $5-30$ & $1-2$ & $3-5$ & $6-30$ & $1-2$ & $3-5$ & $6-30$ \\
\hline 4 (Female) & 0 & $1-3$ & $4-30$ & 0 & $1-5$ & $6-30$ & $1-3$ & $4-5$ & $6-30$ & 0 & $1-3$ & $4-30$ \\
\hline 5 (Female) & $1-2$ & $3-6$ & $7-30$ & 1 & $2-4$ & $5-30$ & $1-4$ & 5 & $6-30$ & $1-3$ & $4-5$ & $6-30$ \\
\hline 6 (Female) & 1 & $2-5$ & $6-30$ & 1 & $2-4$ & $5-30$ & $1-2$ & $3-5$ & $6-30$ & $1-2$ & $3-5$ & 6-30 \\
\hline
\end{tabular}

OP: Obviously present; MP: moderately present; NP: not present. ${ }^{a}$ Eyelid is partially or completely closed. ${ }^{\mathrm{b}}$ Contraction around the muzzle so that the whisker pads are pressed against the side of the face. ${ }^{c}$ Whiskers are straightened and extended horizontally or pulled back toward the cheeks. dEars are rotated away from normal position and tend to move backward and be held closer to the back or side of the body.

Design of the surgical approach. To study by anatomical dissection, the animals were sacrificed with an overdose of sodium pentobarbital by an intravenous line $(90 \mathrm{mg} / \mathrm{kg}$ in weight). The thickness of certain areas of bone was measured with a digital caliper with an accuracy of $0.1 \mathrm{~mm}$. The maxillary sinus is immediately ventral (anterior) to the upper molar region and may be accessed from inside by going through the vestibular bone (Figure 1A). At the anatomical area chosen for the approach, a thin mucosa of lax tissue can be identified, covering the fenestrated cortical bone in which the presence of multiple venous vessels can be observed. Because of its thickness (less than $1 \mathrm{~mm}$ ), rapidly accessing the maxillary sinus with a carefully surgical drilling is possible. The sinus floor is firm and has a variable thickness between 0.8 and $1.4 \mathrm{~mm}$. The mucosa is resistant, and its lift allows a subantral volume of between 0.2 and $0.3 \mathrm{cc}$. As noted, an incision of $15-20 \mathrm{~mm}$ on the boundary between the palatal and buccal mucosa, immediately ventral to the molar region and creating a mucoperiosteal flap, produces less involvement of blood vessels, allowing an approach with better visibility of the vestibular cortical bone. Inside the maxillary sinus, the nasolacrimal duct is visible diagonally along the top of the cavity (Figure $1 \mathrm{~B}$ and C). It is important to consider performing the sinus approach, which should be the most posterior and lowest region immediately anterior of the maxillary molars to achieve the floor lifting of the maxillary sinus and obtain an adequate space to place biomaterial (Figure 1B and C).

Surgical procedure. The rabbits were pre-medicated 1 hour prior to surgery with an oral dose in drops of amoxicillin $50 \mathrm{mg} / \mathrm{kg}$. For sedation of the animals, a dose of $30 \mathrm{mg} / \mathrm{kg}$ ketamine supplemented with $3 \mathrm{mg} / \mathrm{kg}$ xylazine intramuscularly (i.m.) was used. In addition, as a local anesthetic, $0.5 \mathrm{ml}$ of $2 \%$ lidocaine, was applied with a dose of 1:100,000 of epinephrine (Octocaine-100; Novocol Pharmaceutical, Ontario, Canada) in the mucosa of the lateral region of the maxilla at the maxillary sinus level. To maintain the mouth open during the procedure, a silicone stopper of $2.0 \mathrm{~cm}$ was used, which was placed between the maxillary and mandibular incisors.

The intraoral approach to the maxillary sinus was performed using microsurgical instruments (Karl Storz, Tuttlingen, Germany) and with endoscopic assistance through a $2.7-\mathrm{mm}$ optic in a rigid support, with 30 degrees of angulation and optical fiber under continuous irrigation (external and internal). The procedure was performed unilaterally. A $15-$ to $20-\mathrm{mm}$ incision was made in an anteroposterior direction on the boundary between the palatal and the buccal lateral sidewall mucosa, immediately ventral to the molar region and separating the buccal mucosa and periosteum with a beveled periosteal elevator, exposing the uncovered surface (Figure 2A). The cortical bone was removed with a 4-mm diamond burr under permanent irrigation with saline solution (Figure 2B). The sinus membrane (Schneider membrane) was exposed directly (Figure 2C) and was separated with a microsurgical elevator specifically for sinus mucosa type 0 (dish knives) and a microsurgical elevator type $\mathrm{T}$ for tunneling (Figure 2D), creating the subantral space (Figure 2E), where $0.2-0.3 \mathrm{cc}$ of demineralized human bone matrix (DynaGraft, Keystone Dental, Burlington, MA, USA) was placed to achieve augmentation of the maxillary sinus floor (Figure 2F). Finally, the wounds were closed with absorbable suture type Vicryl (Ethicon, Novartis Animal Health US, Inc.; Greensboro, NC, USA) 3-0.

After surgery, an antibiotic therapy with gentamicin $(20 \mathrm{mg} / \mathrm{kg} / \mathrm{day}$ i.m.) and analgesic with sodium diclofenac $(80 \mathrm{mg} / \mathrm{kg} /$ day i.m.) for 3 days were applied in each animal. During the first 10 days after the surgery, the animals were fed a controlled soft diet. Subsequently, computerized evaluation of the maxillary sinus membrane lift was performed through cone-beam computed tomography. All the specimens were observed for a period of 30 days.

Monitoring period. In order to assess the applicability of the described experimental model, a combination of protocols designed to recognize pain, welfare, and stress in experimental animals was applied for 30 days for a single examiner: i) A modification of the protocol proposed by Morton and Griffiths (10), which included weekly weight assessment and the observation of various behavioral aspects such as food consumption, volume of stools, natural behavior, and behavior to stimuli; ii) The Rabbit Grimace Scale (11-12), which is composed of five facial action units: orbital tightening, cheek flattening, nose shape, whisker position and ear position. Furthermore, visual examination of the surgical zone was performed to assess the volume increase, bleeding, suture stability, and possibility of infection. Finally, the results were compared with a control group consisting of three Californian rabbits without surgical procedure (control), maintained under the same observation conditions and for the same period as the experimental group. 

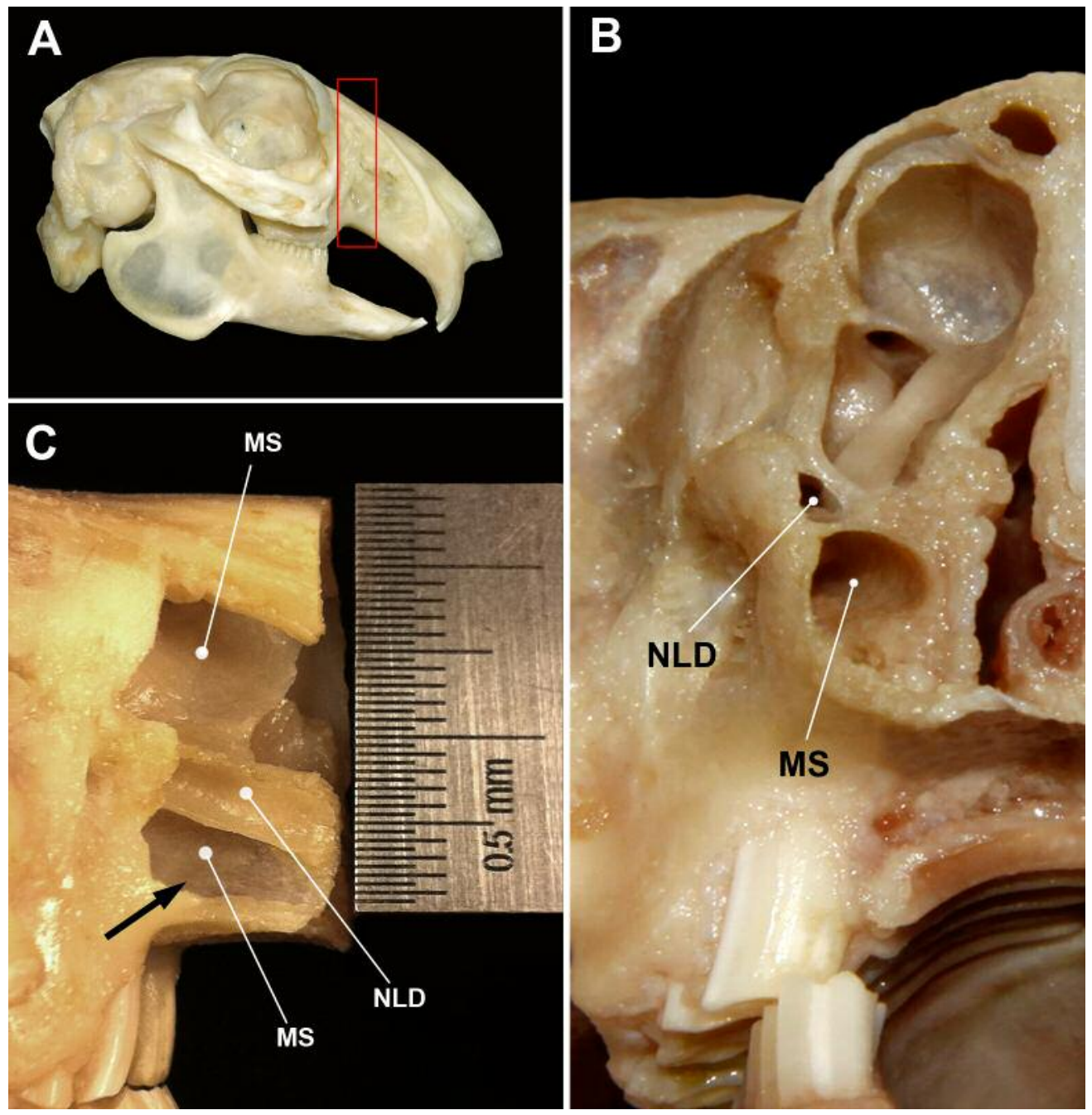

Figure 1. Anatomical study for access to the maxillary sinus. A: The box indicates the bone area of intervention. B: Frontal section of the nasal region at the level of the box shown in A. The bone structure with mucosa of airway, as well as the maxillary sinus (MS), and nasolacrimal duct $(N L D)$ can be observed. C: The lateral view of the maxillary sinus, with the nasolacrimal duct exposed. The space indicated by the arrow was used for biomaterial placement after the surgical creation of a subantral space by endoscope-assisted intraoral access.

\section{Results}

The SFA methodology described in this study proved to be minimally invasive, allowing a general uneventful recovery for all rabbits. In all the specimens, application of $0.2-0.3 \mathrm{cc}$ of biomaterial in the subantral space created was achieved. The image analyses of coronal sections obtained using conebeam computed tomography showed a minor volume of maxillary sinus because of the membrane lift from the floor (Figure 3). During the postoperative observation period, animals fed normally. The parameters studied following the proposal of Morton \& Griffiths and the Rabbit Grimace Scale showed no significant alterations to suggest a need to suspend the experimental protocol. Considering the initial weight of each specimen, no significant changes in weight over the period of 30 days were observed. The number and volume of stools did not significantly differ compared to the control group. The natural behavior and response to stimuli was considered different only in the first week after the surgical procedure compared with the control group. Table I details the results of recording using the Rabbit Grimace Scale. Due to the difficulty in recording the nose shape, this aspect was not included in the Table. At local examination, all cases showed localized edema that remained during the first 2-3 days.

Tissue healing was normal without alterations in the shape. No sutures were observed after 30 days in any specimen. 


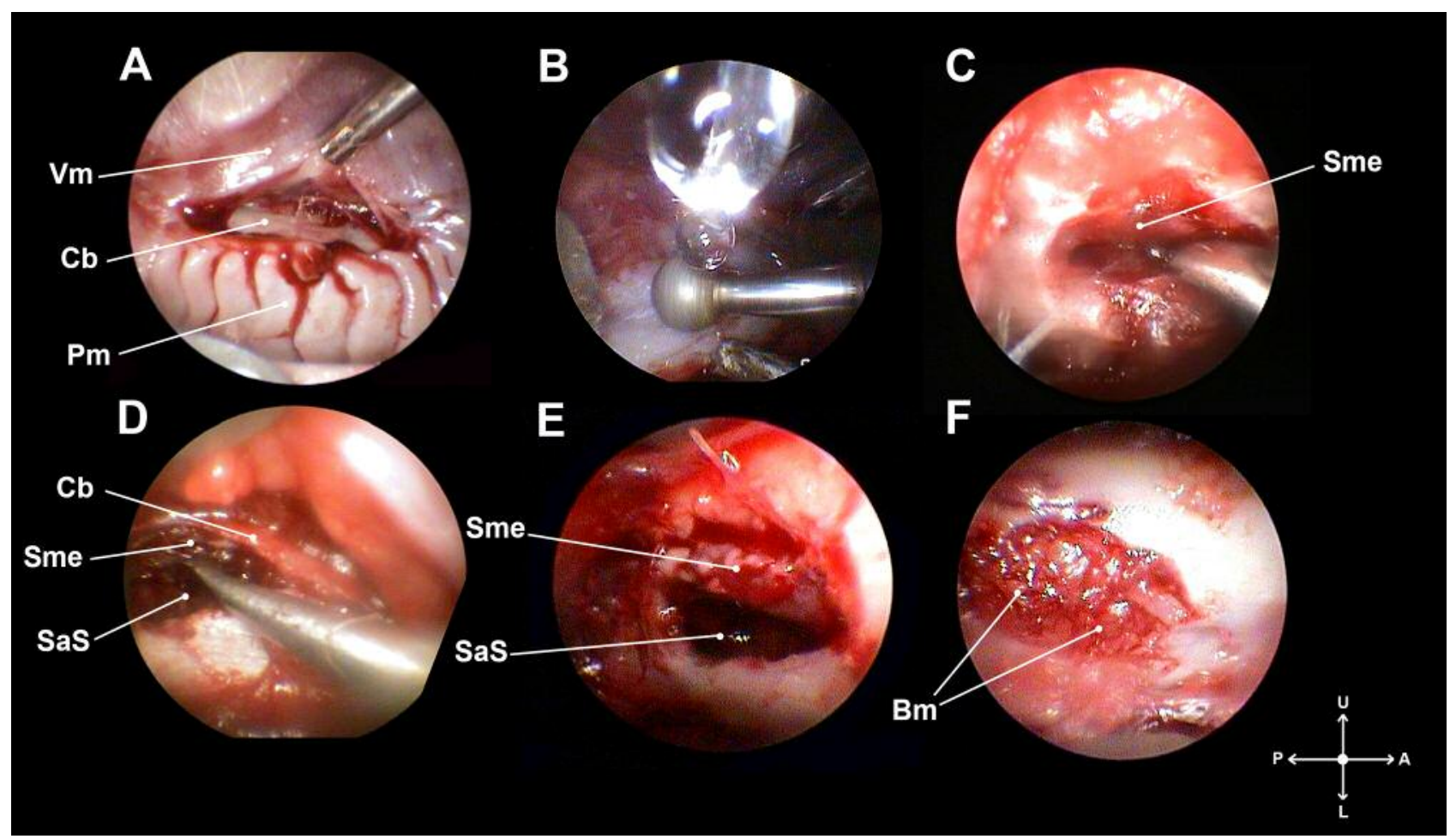

Figure 2. Sequence of biomaterial placement in a subantral space for maxillary sinus floor augmentation in a rabbit with endoscopic assistance. A: Initial incision about $2 \mathrm{~cm}$ on the boundary between the palatal $(\mathrm{Pm})$ and vestibular mucosa $(\mathrm{Vm})$. In B: The vestibular bone was milled with a surgical bur to access inside the maxillary sinus. C-E: Membrane elevation and creation of a subantral space (SaS). F: Insertion of biomaterial $(B m)$ in the subantral space. A: Anterior; Cb: cortical bone; L: lower; P: posterior; Sme: sinus membrane; U: upper.

\section{Discussion}

Various experimental techniques for surgical access to the maxillary sinus have been developed to allow bone thickness to be increased through the placement of biomaterials using rabbits as an experimental model. This model has various advantages because of its easy handling and observation, and is widely adopted by the scientific community in bioethics, allowing a standardization of the environment and genetic normalization. Therefore, it is an appropriate and feasible model for experimental studies in medical and dental sciences (13). The extraoral approach technique is the most used in rabbits. It describes a skin incision in the center of the nasal dorsum. Then the muscle tissue and periosteum are dissected to expose the nasal bone and the nasoincisal suture, which is used as a reference point. Over this surface, a procedure producing a unilateral or bilateral window shape is performed in the bone from where it can separate the maxillary sinus mucosa of the bone for biomaterial placement $(4,6,7,14-17)$. The main disadvantage of this technique concerns the means of separating the sinus membrane from the bone wall. Specifically, the SFA or sinus membrane should not strictly be elevated. Furthermore, this technique can produce an inner growth and invasion of fibrous connective tissue to the bone window and sinus antrum. This can be overcome by placing barriers such as collagen membranes $(14,17)$, which is a more complex and expensive procedure. Moreover, this access increases the risk of trauma and damage to the sinus membrane, as it should be pushing internally, separating the lateral and medial wall and eventually reaching and raising the maxillary sinus floor.

Others have described an extraoral access in the rabbit by an incision from the skin of the maxillary region, dividing the subcutaneous tissues and masseter muscle to expose the maxillary periosteum $(8,18,19)$. Then the periosteum is separated and elevated dorsally, exposing the maxillary sinus lateral bone wall, where the surgical window that allows the observation and elevation of the sinus membrane directly from the floor is performed. Although this technique has achieved success in increasing the alveolar bone height, the surgical trauma due to the need to dissect several anatomical planes must be considered. Allegrini et al. (2) described that the rupture and subsequent suture of all these planes increases the risks of infection, pain, inflammation, and slow 


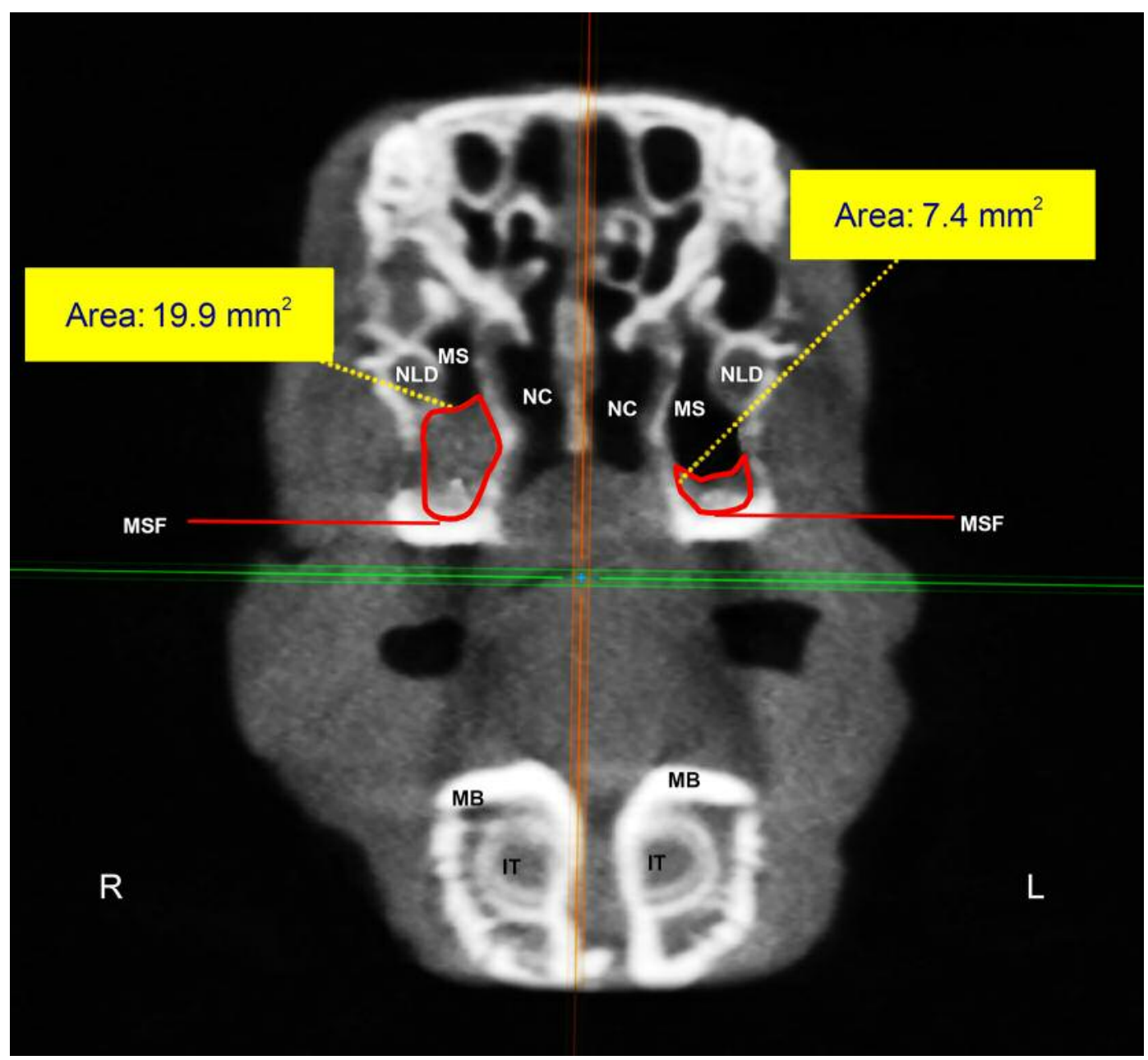

Figure 3. Cone-beam computed tomographic image of a coronal section of the head from an experimental rabbit that shows the maxillary sinuses. The bimaxillary analyses showed a decrease in volume of the right sinus cavity because of the sinus floor augmentation procedure. IT: Incisive teeth; L: left side; MB: mandibular bone; MS: maxillary sinus; MSF: maxillary sinus floor; NC: nasal cavity; NLD: nasolacrimal duct; R: right side.

healing. To this drawback, the reports of moderate increases in bone volume in the surgical area are added, probably because of edema caused by trauma and a comprehensive approach and pain and bleeding from the surgical site (1, 15). Rahmani et al. (20) reported that extraoral access from the masseteric region generates complications, such as respiratory distress of the animals, because of congestion of the trachea and partial obstruction caused by fibrin clots.

In humans, unlike most animal models used, maxillary SFA is performed by an intraoral approach $(21,22)$. Rahmani et al. (20) described an intraoral approach in rabbits from the lining of the alveolar ridge under the maxillary sinus at a distance of $4 \mathrm{~mm}$ ventral to the maxillary first molar to allow a full-thickness mucoperiosteal flap elevation, exposing the bone and performing osteotomy. This approach is particularly attractive and has advantages, such as similarity to what has been done in humans. This leads to less local trauma, due dissecting fewer anatomical planes in order to access the bone tissue, and direct access to the sinus floor, homologous to the procedure performed in humans that does not require access through the skin or cut muscle tissue but only through the mucosa, periosteum, and bone. However, a major limitation of this model is the small size of the oral cavity of the rabbit, where it is difficult to achieve optimal visualization to ensure proper placement. In this sense, endoscopy offers an appropriate tool for improving the surgical technique. New applications have been reported in oral surgery, maxillofacial surgery, and implantology (23-25). In SFA, endoscopic assistance optimizes the success of an intraoral surgical approach. This technology optimizes viewing, achieving up 
to $4 \times$ magnification. During surgery, it achieves a minimally invasive approach with a mucosal incision no longer than 2 $\mathrm{cm}$, with a reduction in the number of tissue planes involved (compared with the extraoral approach technique) and little bleeding. Furthermore, the optical fiber of the endoscope provides an additional light source for the system, allowing excellent handling of the surgical site. This facilitates the membrane lift and also allows good visualization for optimal positioning and packaging of the biomaterial to be used. Moreover, the optical fiber requires a maxillary sinus bone window access with no more than $3 \mathrm{~mm}$ in diameter. This causes less local bone trauma and is in accordance with some authors as Sohn et al. $(4 \times 8 \mathrm{~mm}) 4)$, Xia et al. $(5 \times 10 \mathrm{~mm})$ (15) and Hou et al. (4×4 mm) (17).

One of the most important anatomical limitations of the technique is the mouth opening of the rabbit, which is a difficult surgical action. However, the use of a stabilizer stopper made of silicone expedited the handling of the endoscopic optics and supported the surgical site without complications. It is also important to consider the expensive cost of endoscopy, a trained operator and microsurgical instruments necessary for the procedure as limitations. However, the refinement achieved allows several advantages: Fewer specimens need to be used in experiments, adequate dosage of biomaterial application, similar functional conditions, and the opportunity of using various types of bone biomaterials in other studies.

In recent years, various biomaterials for SFA have been developed. Therefore, using a refined experimental methodology is highly relevant. The experimental model protocol proposed here proved to be suitable as shown from the surgical, short-term measurable results according to the objectives of our study, to which we add the ease of recording the results through histomorphometry and imaging techniques.

As in humans, surgery in rabbits should be as least traumatic as possible. A minimally invasive approach improves all experimental parameters, diminishing biases of the surgical procedure to facilitate the maintenance and recovery of the animal model. When considering the guidelines proposed by the 3Rs of Russell and Burch (replacement, reduction, and refinement) $(26,27)$, this protocol reduces morbidity and mortality of animals because of its conservative design, which conditions the tissue for a speedy recovery, thus improving the postoperative period; it also optimizes dosage and application of biomaterials for maxillary SFA in experimental purposes. In conclusion, this new access model for SFA in rabbits is an option that improves the predictability of the outcome and diminishes the bias in the use of experimental models by endoscopic assistance.

\section{Conflicts of Interest}

The Authors declare that no conflicts of interest exist regarding this study.

\section{Authors' Contributions}

IVG, VB and WE designed and planned the study; IVG, VB and EB performed the animal experiments; EB realized photographic register during experiment; IVG, WAR, OAA and NFV researched and wrote the article; WAR and OAA organized and edited images; WE and VB offered scientific advice and proofread the article. All Authors read and approved the article.

\section{Acknowledgements}

This study was economically supported $(70 \%)$ by the Faculty of Dentistry of the University of La Frontera and $30 \%$ by MINEDUCUA project, code ANT 1855 and the Program of Research Assistant 2019, University of Antofagasta.

\section{References}

1 Wada K, Niimi A, Watanabe K, Sawai T and Ueda M: Maxillary sinus floor augmentation in rabbits: A comparative histologichistomorphometric study between rhBMP-2 and autogenous bone. Int J Periodontics Restorative Dent 21(3): 252-263, 2001. PMID: 11490402.

2 Allegrini SJr, Yoshimoto M, Salles MB and Konig BJr: The effects of bovine BMP associated to HA in maxillary sinus lifting in rabbits. Ann Anat 185(4): 343-349, 2003. PMID: 12924472. DOI: 10.1016/S0940-9602(03)80056-0

$3 \mathrm{Xu} \mathrm{H}$, Shimizu Y, Onodera K and Ooya K: Long-term outcome of augmentation of the maxillary sinus using deproteinised bone particles experimental study in rabbits. Br J Oral Maxillofac Surg 43(1): 40-45, 2005. PMID: 15620773. DOI: 10.1016/ j.bjoms.2004.10.004

4 Sohn DS, Kim WS, An KM, Song KJ, Lee JM and Mun YS: Comparative histomorphometric analysis of maxillary sinus augmentation with and without bone grafting in rabbit. Implant Dent 19(3): 259-270, 2010. PMID: 20523182. DOI: 10.1097/ ID.0b013e3181df1406

5 Watanabe K, Niimi A and Ueda M: Autogenous bone grafts in the rabbit maxillary sinus. Oral Surg Oral Med Oral Pathol Oral Radiol Endod 88(1): 26-32, 1999. PMID: 10442941. DOI: 10.1016/S1079-2104(99)70189-7

6 Asai S, Shimizu Y and Ooya K: Maxillary sinus augmentation model in rabbits: effect of occluded nasal ostium on new bone formation. Clin Oral Implants Res 13(4): 405-409, 2002. PMID: 12175378. DOI: 10.1034/j.1600-0501.2002.130409.x

7 Ho SK, Peel SA, Hu ZM, Sándor GK and Clokie CM: Augmentation of the maxillary sinus: comparison of bioimplants containing bone morphogenetic protein and autogenous bone in a rabbit model. J Can Dent Assoc 76: a108, 2010. PMID: 21044421.

8 Hu Z, Peel SA, Ho SK, Sándor GK, Su Y and Clokie CM: The expression of bone matrix proteins induced by different bioimplants in a rabbit sinus lift model. J Biomed Mater Res A 95(4): 1048-1054, 2010. PMID: 20878983. DOI: 10.1002/ jbm.a.32911

9 National Research Council (US) Committee for the Update of the Guide for the Care and Use of Laboratory Animals. Guide for the Care and Use of Laboratory Animals. Eighth edition. Washington (DC): National Academies Press (US); 2011. 
10 Morton DB and Griffiths PH: Guidelines on the recognition of pain, distress and discomfort in experimental animals and an hypothesis for assessment. Vet Rec 116(16): 431-436, 1985. PMID: 3923690. DOI: 10.1136/vr.116.16.431

11 Keating SC, Thomas AA, Flecknell PA, Leach MC: Evaluation of EMLA cream for preventing pain during tattooing of rabbits: Changes in physiological, behavioural and facial expression responses. PLoS One 7(9): e44437, 2012. PMID: 22970216. DOI: 10.1371 journal.pone.0044437

12 Hampshire V, Robertson S: Using the facial grimace scale to evaluate rabbit wellness in post-procedural monitoring. Lab Anim 44(7): 259-260, 2015. PMID: 26091129. DOI: 10.1038/laban.806

13 Calasans-Maia MD, Monteiro ML, Áscoli FO and Granjeiro JM: The rabbit as an animal model for experimental surgery. Acta Cir Bras 24(4): 325-328, 2009. PMID: 19705034. DOI: 10.1590/S0102-86502009000400014

14 Riecke B, Heiland M, Hothan A, Morlock M, Amling M and Blake FA: Primary implant stability after maxillary sinus augmentation with autogenous mesenchymal stem cells: a biomechanical evaluation in rabbits. Clin Oral Implants Res 22(11): 1242-1246, 2011. PMID: 21504476. DOI: 10.1111/ j.1600-0501.2010.02043.x

15 Xia L, Xu Y, Chang Q, Sun X, Zeng D, Zhang W, Zhang X, Zhang $\mathrm{Z}$ and Jiang $\mathrm{X}$ : Maxillary sinus floor elevation using Bmp2 and Nell1 gene-modified bone marrow stromal cells and TCP in rabbits. Calcif Tissue Int 89(1): 53-64, 2011. PMID: 21584647. DOI: $10.1007 / \mathrm{s} 00223-011-9493-1$

16 Chaves MD, de Souza Nunes LS, de Oliveira RV, Holgado LA, Filho HN, Matsumoto MA and Ribeiro DA: Bovine hydroxyapatite (Bio-Oss $\left.\left({ }^{\circledR}\right)\right)$ induces osteocalcin, RANK-L and osteoprotegerin expression in sinus lift of rabbits. J Craniomaxillofac Surg 40(8): e315-320, 2012. PMID: 22513050. DOI: $10.1016 / \mathrm{j} . j \mathrm{jcms} .2012 .01 .014$

17 Hou CJ, Liu JL, Li X and Bi LJ: Insulin promotes bone formation in augmented maxillary sinus in diabetic rabbits. Int J Oral Maxillofac Surg 41(3): 400-407, 2012. PMID: 22099315. DOI: $10.1016 /$ j.ijom.2011.10.004

18 Trbakovic A, Hedenqvist P, Mellgren T, Ley C, Hilborn J, Ossipov D, Ekman S, Johansson CB, Jensen-Waern M, Thor A: A new synthetic granular calcium phosphate compound induces new bone in a sinus lift rabbit model. J Dent 70: 31-39, 2018. PMID: 29258851. DOI: 10.1016/j.jdent.2017.12.009
19 Kim YS, Kim SH, Kim KH, Jhin MJ, Kim WK, Lee YK, Seol YJ, Lee YM: Rabbit maxillary sinus augmentation model with simultaneous implant placement: differential responses to the graft materials. J Periodontal Implant Sci 42(6): 204-211, 2012. PMID: 23346463. DOI: 10.5051/jpis.2012.42.6.204.

20 Rahmani M, Shimada E, Rokni S, Deporter DA, Adegbembo AO, Valiquette $\mathrm{N}$, and Pilliar RM: Osteotome sinus elevation and simultaneous placement of porous-surfaced dental implants: A morphometric study in rabbits. Clin Oral Implants Res 16(6): 692-699, 2005. PMID: 16307576. DOI: 10.1111/j.16000501.2005.01163.x

21 Boyne PJ and James RA: Grafting of the maxillary sinus floor with autogenous marrow and bone. J Oral Surg 38(8): 613-616, 1980. PMID: 6993637.

22 Tatum H Jr: Maxillary and sinus implant reconstructions. Dent Clin North Am 30(2): 207-229, 1986. PMID: 3516738.

23 Engelke W, Beltrán V: Endoscopic approach to remove a dental implant with simultaneous laser-cystectomy. J Craniofac Surg 30(1): e11-e12, 2019. PMID: 30376504. DOI: 10.1097/ SCS.0000000000004874

24 Engelke W and Capobianco M: Flapless sinus floor augmentation using endoscopy combined with CT scan-designed surgical templates: Method and report of 6 consecutive cases. Int J Oral Maxillofac Implants 20(6): 891-897, 2005. PMID: 16392346.

25 Beltrán V, Fuentes R and Engelke W: Endoscopic visualization of anatomic structures as a support tool in oral surgery and implantology. J Oral Maxillofac Surg 70(1): e1-6, 2012. PMID: 22182657. DOI: $10.1016 /$ j.joms.2011.09.011

26 Russell WMS and Burch R: The principles of humane experimental technique. London, UK: Methuen, 1959.

27 Tannenbaum J and Bennett BT: Russell and Burch's 3Rs then and now: The need for clarity in definition and purpose. J Am Assoc Lab Anim Sci 54(2): 120-132, 2015. PMID: 25836957.
Received August 5, 2019

Revised August 27, 2019

Accepted September 3, 2019 